\title{
ESTUDO DO EFEITO DE MATERIAL PARTICULADO NA FORMAÇÃO DA TORTA DE FILTRAÇÃO E NA INVASÃO DE FILTRADO
}

\author{
A. S. F. S. FERRAZ ${ }^{1}$, M. F. M. NOGUEIRA ${ }^{1}$, L. A. CALÇADA ${ }^{1}$, C. M. SCHEID ${ }^{1}$, S. C. \\ MAGALHAES Filho $^{1}$, E. C. H. PARAISO ${ }^{1}$, A.T. A. WALDMANN ${ }^{2}$ \\ ${ }^{1}$ Universidade Federal Rural do Rio de Janeiro, Departamento de Engenharia Química \\ ${ }^{2}$ Cenpes/Petrobras, Centro de Pesquisas Leopoldo Américo Miguez de Mello, Rio de Janeiro, \\ Brazil \\ calcada@ufrrj.br
}

\begin{abstract}
RESUMO: Partículas sólidas são usadas como adensante e material obturante na formulação de fluidos de perfuração. As características físico-químicas destes materiais têm forte influência na formação da torta e consequentemente na taxa de filtração e invasão de filtrado nas formações rochosas. A invasão indesejável pode causar danos irreversíveis à formação. Desta forma, o controle das propriedades da torta é fundamental no processo de perfuração de poços de petróleo. Neste trabalho, objetivou-se estudar o efeito da forma e da distribuição granulométrica de carbonato de cálcio e da esfera de vidro na taxa de filtração estática. Os experimentos foram realizados em uma célula high temperature/high presssure (HTHP) para a filtração estática e dinâmica em condições compatíveis com aquelas encontradas durante a perfuração de poços de petróleo. Concluiu-se que quando é utilizado um fluido não newtoniano ocorre a influência no volume de filtrado devido à interação da granulometria do carbonato de cálcio e goma xantana.
\end{abstract}

\section{INTRODUÇÃO}

Em condições overbalance na perfuração dos poços de petróleo, à medida que a broca penetra a rocha reservatório, o fluido de perfuração invade a formação devido ao diferencial de pressão positivo entre o mesmo e o fluido do reservatório (Civan, 2007). Porções do fluido são perdidas para as formações adjacentes, enquanto parte dos sólidos presentes no fluido de perfuração, constituída por partículas menores que os poros da formação, penetra na rocha durante a perda do fluido, tamponando rapidamente a região ao redor do poço. Já as partículas maiores acumulam-se na parede do poço, iniciando a formação do reboco externo.

Neste trabalho, foi estudado o efeito da distribuição granulométrica do carbonato de cálcio na taxa de filtração. Foi utilizado um único tipo de carbonato de cálcio e separado em três faixas granulométricas. Cada faixa granulométrica foi utilizada para preparar o fluido de perfuração, sendo todos fluidos base água. Os fluidos foram filtrados sob condição estática de operação. 


\section{9 a 22 de outubro de 2014 \\ Florianópolis/SC}

A filtração estática ocorre quando a circulação é interrompida, o reboco de baixa permeabilidade formado cresce continuamente sendo o responsável por controlar as taxas de filtração no poço. Portanto, na medida em que a espessura do reboco vai aumentando, as taxas de filtração vão diminuindo ao longo do tempo.

Segundo Civan (2007), a invasão de partículas sólidas ocorre principalmente durante o spurt loss, uma vez que torta encontra-se em formação, um número pequeno de partículas é capaz de invadir a rocha e a consequente retenção das partículas pela malha sinuosa que o meio apresenta. Estudos realizados por Bailey et al. (2000), mostram uma forte correlação entre invasão e dano, e que partícula maiores reduzem o sport loss. A avaliação das propriedades filtrante do fluido de perfuração é normalmente realizada através de ensaios de filtração estática e dinâmica em meios porosos. Nestes ensaios, foram colocados um volume fixo de fluido na célula de filtração HTHP e a mesma se da sobre um meio filtrante pré-saturado. $\mathrm{O}$ volume de fluido que atravessa o meio poroso é monitorado ao longo do tempo.

\section{REVISÃO DE LITERATURA}

\subsection{Efeitos da distribuição da partícula}

Os principais fatores que determinam o dano nas formações dos poros devido à invasão de sólidos são: distribuição do tamanho de partícula, permeabilidade da rocha, distribuição de tamanho dos poros, concentração de sólidos do fluido de perfuração, taxa de circulação de fluido de perfuração e o comportamento reologico (Chesser, et al., 1994).

Embora alguma invasão dos sólidos e dano a formação são inerentes a todo o processo de perfuração, é possível minimizar os mesmos causados pela invasão de sólidos, e a profundidade dos danos causados, pelo correto dimensionamento da distribuição das partículas fluido de perfuração.

Segundo Gallino et al. (2001) a distribuição do tamanho de partícula do fluido de perfuração é um parâmetro físico importante, juntamente com outras propriedades, tais como a densidade e reologia.

Gates \& Bowie (1942) estudaram a relação entre a distribuição de tamanho da partícula e as propriedades de filtração. Os autores concluiram que a maioria dos fluidos de perfuração que tiveram as taxa de filtração reduzida foi composto por fluidos com distribuição de partículas relativamente uniforme.

Kumbein \& Monk (1943) investigaram os efeitos da distribuição do tamanho de partícula na permeabilidade do reboco externo. Seus resultados mostraram que a permeabilidade decresce com: A diminuição do diâmetro médio de partícula; A polidispersão do agente adensante; 0 excesso de partículas finas na distribuição granulométrica. 


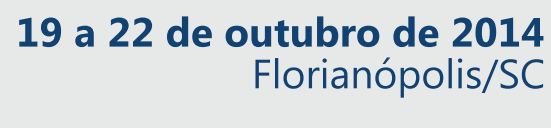

\section{MATERIAL E MÉTODOS}

Os experimentos foram realizados em uma célula high temperature/high presssure (HTHP) para a filtração estática, utilizando filtro de papel da Marca Fann como meio filtrante. O papel de filtro tem diâmetro de 6,30 cm e retenção de particulas de 2,7-5,0 $\mu \mathrm{m}$. Os ensaios medem o volume de filtrado em função do tempo. Todos os experimentos foram feitos com pressão controlada. A condição de operação estática foi realizada durante 1 hora de filtração, sendo que em 30 minutos foram aplicados uma pressão de 500 psi e nos demais 30 minutos uma pressão de 1000 psi.

\subsection{Seleção do carbonato de cálcio}

A seleção do carbonato de cálcio da empresa BRASILMINAS foi baseada na escolha de um material com uma larga faixa de distribuição granulométrica. Inicialmente foi feita uma separação mecânica com um agitador eletromagnético de peneiras BERTEL. O material foi separado em três grupos com classificação granulométrica distinta. Para a caracterização final da distribuição granulométrica foi utilizado um analisador de partículas MALVERN (MASTERSIZER 2000 HYDRO), que utiliza a técnica de difração a laser para determinação de distribuição de tamanho de partículas.

\subsection{Fluido de perfuração}

Objetivando verificar a influência do tamanho das partículas do carbonato de cálcio na formação da torta de filtração e invasão de filtrado foram prepados vários tipos de fluidos. Além das partículas de carbonato de cálcio foram preparados fluidos com esfera de vidro fornecido pela empresa POTTERS que possuem distribuição granulométrica mais restrita. Os fluidos foram preparados no agitador Hamilton Beach, seguindo a formulação da Tabela 1.

Tabela 1- Formulação dos fluidos

\begin{tabular}{|c|c|c|c|c|c|c|c|c|c|c|}
\hline Componetes & Tipo 1 & Tipo 2 & Tipo 3 & Tipo 4 & Tipo 5 & Tipo 6 & Tipo 7 & Tipo 8 & Tipo 9 & Tipo 10 \\
\hline Água (L) & 0,5 & 0,5 & 0,5 & 0,025 & 0,025 & 0,025 & 0,5 & 0,5 & 0,025 & 0,025 \\
\hline Goma Xantana (lb/bbl) & 2,85 & 2,85 & 2,85 & - & - & - & 2,85 & 2,85 & - & - \\
\hline Glicerina (L) & - & - & - & 0,475 & 0,475 & 0,475 & - & - & 0,475 & 0,475 \\
\hline $\mathrm{CaCO}_{3}(0-53 \mu \mathrm{m})$ & 137,25 & - & - & 137,25 & - & - & - & - & - & - \\
\hline $\mathrm{CaCO}_{3}(53-106 \mu \mathrm{m})$ & - & 137,25 & - & - & 137,25 & - & - & - & - & - \\
\hline $\mathrm{CaCO}_{3}(106-150 \mu \mathrm{m})$ & - & - & 137,25 & - & _ & 137,25 & - & - & - & - \\
\hline Esfera de vidro $(-75 \mu \mathrm{m})$ & - & - & - & - & - & - & 145,83 & - & 145,83 & - \\
\hline Esfera de vidro $(-300+180 \mu \mathrm{m})$ & - & - & - & - & - & - & - & 145,83 & - & 145,83 \\
\hline
\end{tabular}

\subsection{Unidade experimental}

A Figura 1a apresenta a célula de filtração, a Figura 2b apresenta o detalhamento da célula. Trata-se de uma célula HTHP (High temperatura, high pressure), produzida pela OFI Testing Equipments (OFITE). A célula é capaz de realizar medidas das propriedades de filtração sob diferentes condições estáticas e dinâmicas, assemelhando-se as condições operacionais de perfuração de poços de petróleo. A Figura 1c ilustra a interface do sistema de aquisição de dados. O Software faz 
aquisição dos dados de volume de filtrado em função do tempo de filtração. Detalhe do procedimento experimental em Calabrez (2013).

(a)

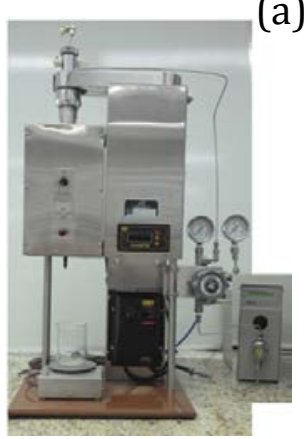

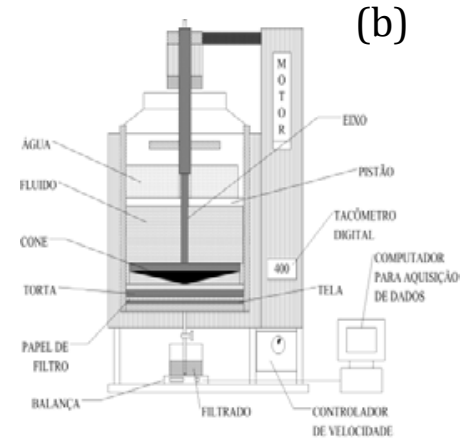

(b)

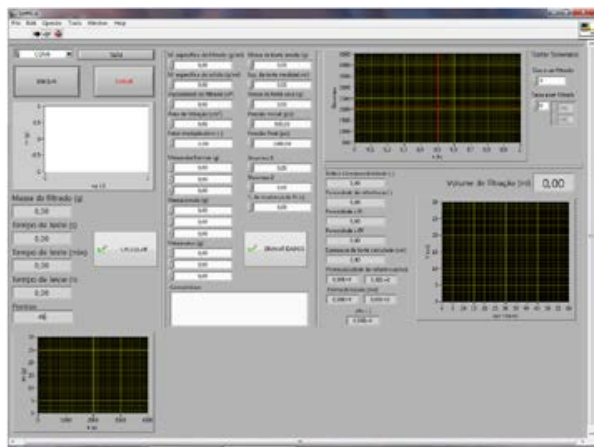

Figura 1- (a) Foto da Célula de filtração HTHP - Marca OFITE; (b) Célula de filtração em detalhes; (c) Software de aquisição de dados.

\section{RESULTADOS E DISCUSSÃO}

\subsection{CARACTERIZAÇÃO DE TAMANHO DAS PARTÍULAS}

As amostras de carbonato de cálcio utilizadas foram separadas no agitador eletromagnético nas faixas de 0-53; 53-106 e 106-150 micrometros. A Figura 2a ilustra a distribuição granulométrica dos três grupos de carbonato de cálcio. A análise com as esferas de vidro são apresentadas na Figura 2b. Ambas as análises realizadas no analisador de partículas Malvern Mastersizer. A Tabela 2 apresenta de forma resumida os parâmetros relevantes para a caracterização dos sólidos: $\mathrm{D}_{10}, \mathrm{D}_{50}, \mathrm{D}_{90}$, sendo: $\mathrm{D}_{10} \mathrm{o}$ diâmetro máximo apresentado por $10 \%$ das partículas $(\mu \mathrm{m}) ; \mathrm{D}_{50} \mathrm{o}$ diâmetro máximo apresentado por $50 \%$ das partículas $(\mu \mathrm{m})$ e $\mathrm{D}_{90}$ o diâmetro máximo apresentado por $90 \%$ das partículas $(\mu \mathrm{m})$.

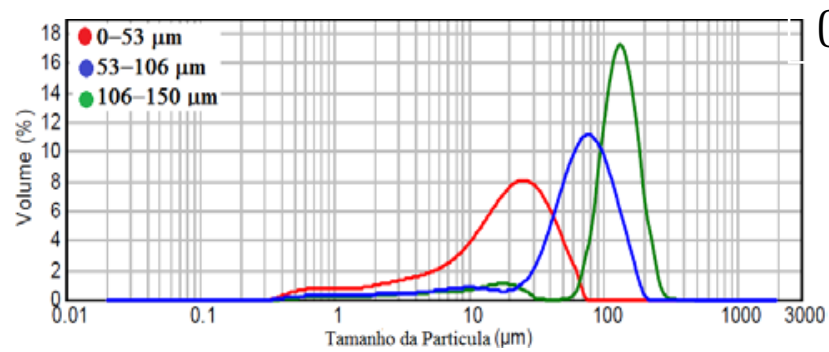

(a)

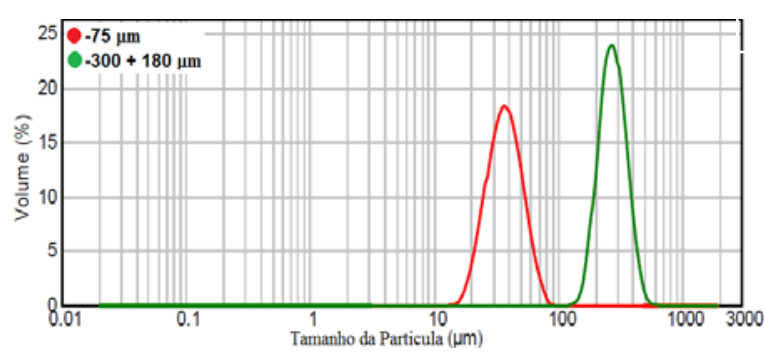

Figura 2- (a) Distribuição granulométrica carbonato de cálcio; (b) Distribuição granulométrica esfera de vidro.

O carbonato de cálcio foi caracterizado pela técnica de microscopia eletrônica de varredura (MEV). As imagens obtidas com o MEV são apresentadas na Figura 3a, b e c. As imagens foram feitas através do shape, que basicamente é um microscópio acoplado ao analisador de partículas e a 
ampliação utilizada para todas as amostras foi de 10x. Vale ressaltar que os resultados apresentados indicam, de maneira qualitativa, a geometria do material.

Tabela 2- Faixa granulométrica dos sólidos adensante.

\begin{tabular}{ccccc}
\hline Sólido adensante & $\mathbf{D}_{\mathbf{1 0}(\boldsymbol{u m})}$ & $\mathbf{D}_{\mathbf{5 0}(\boldsymbol{u m})}$ & $\mathbf{D}_{\mathbf{9 0}(\boldsymbol{u m})}$ & Diâmetro Médio \\
\hline Carbonato de cálcio $(0-53 \mu \mathrm{m})$ & 3,62 & 23,63 & 58,64 & 28.24 \\
Carbonato de cálcio $(53-106 \mu \mathrm{m})$ & 6,61 & 54,51 & 105,61 & 55.36 \\
Carbonato de cálcio $(106-150 \mu \mathrm{m})$ & 23,94 & 132,76 & 236,74 & 139,42 \\
Esfera de vidro $-\mathbf{7 5}(\mu \mathrm{m})$ & 24,45 & 36,97 & 55,79 & 39,02 \\
Esfera de vidro $-300+180(\mu \mathrm{m})$ & 200,01 & 274,55 & 376,31 & 283,62 \\
\hline
\end{tabular}
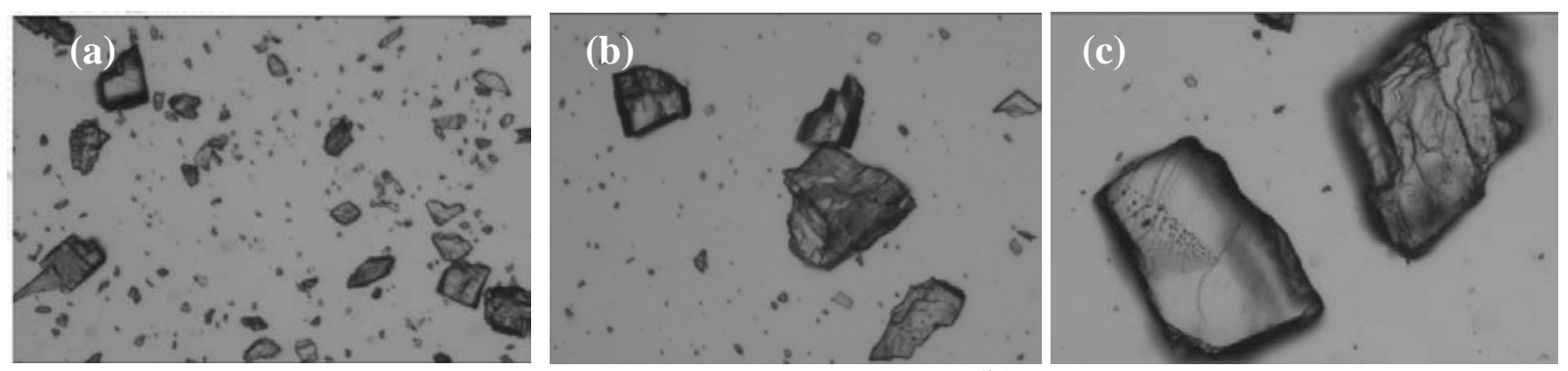

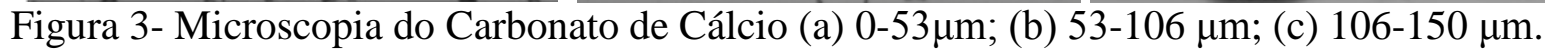

\subsection{Processo de Filtração}

Os experimentos de filtração avaliam o efeito do diâmetro das partículas e do comportamento reológico do fluido sobre o volume de filtrado e as propriedades da torta.

\subsubsection{Experimentos com esfera de vidro e glicerina}

Neste tópico a esfera de vidro foi substituída pelo carbonato de cálcio que é um adensante largamente utilizada em fluidos de perfuração. Além disso utilizou-se a glicerina em substituição a goma xantana, com intuito de retirar a efeito da não-newtoneidade do fluido. Os experimentos realizados com esfera de vidro e glicerina permitiram analisar o efeito do diâmetro das partículas sobre o volume de filtrado de um fluido newtoniano. A Figura 4 mostra as curvas de volume de filtrado versus tempo para o fluido preparado com esfera de vidro de -75 micra e $-300+180$ micra.

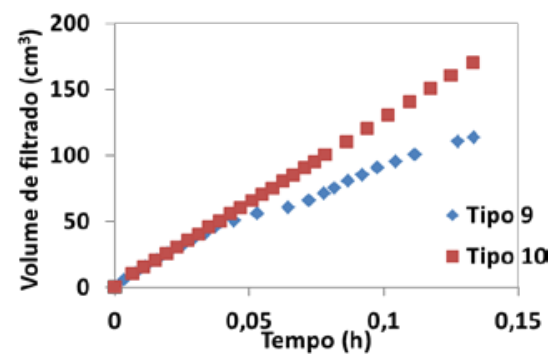

Figura 4- Curva de filtração estática - Tipo 9 e 10. 
Analisando a Figura 4, o fluido (tipo 9) preparado com esfera de vidro -75 micra produz um menor volume de filtrado quando comparado com o fluido (tipo 10) de esfera de vidro -300 +180 micra. Pode-se observar que para um fluido newtoniano o volume de filtrado aumenta com a maior granulometria

\subsubsection{Experimento com carbonato de cálcio e glicerina}

Os experimentos realizados com carbonato de cálcio e glicerina permitiram analisar o comportamento da filtração sem o efeito da não- newtoneidade causada pela goma xantana, que é utilizada para manter as partículas em suspensão. A Figura 5a apresenta o reograma do fluido analisado e Figura 5b a curva de filtração do fluido.

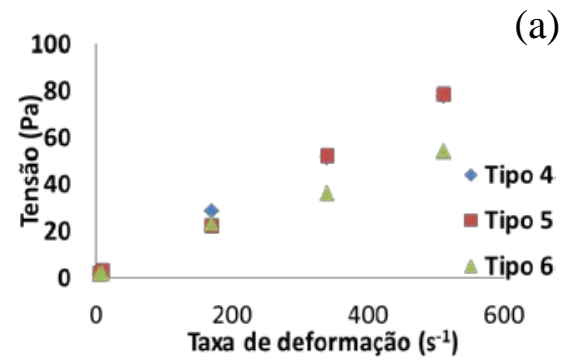

(a)

Figura 5- (a) Reograma dos fluidos. (b) Curva de filtração estática - Tipo 4, 5 e 6. (b)

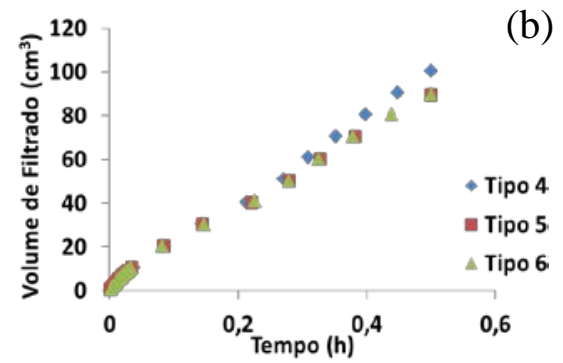

Analisando os resultados do experimento, é possivel perceber que a alteração do tamanho das particulas causou uma leve alteração na viscosidade aparente da amostra. Além disso, o uso da glicerina em substituição a goma xantana causou uma aproximação nas curvas de filtração.

\subsubsection{Experimentos com esfera de vidro e goma xantana}

Com o objetivo de avaliar o efeito do tamanho da partícula na filtração, usou-se esfera de vidro com faixa de distribuição granulométrica mais estreita, sem sobreposição das partículas e o efeito da goma xantana. A Figura 6a apresenta o reograma do fluido analisado e Figura 6b curva de filtração do fluido.
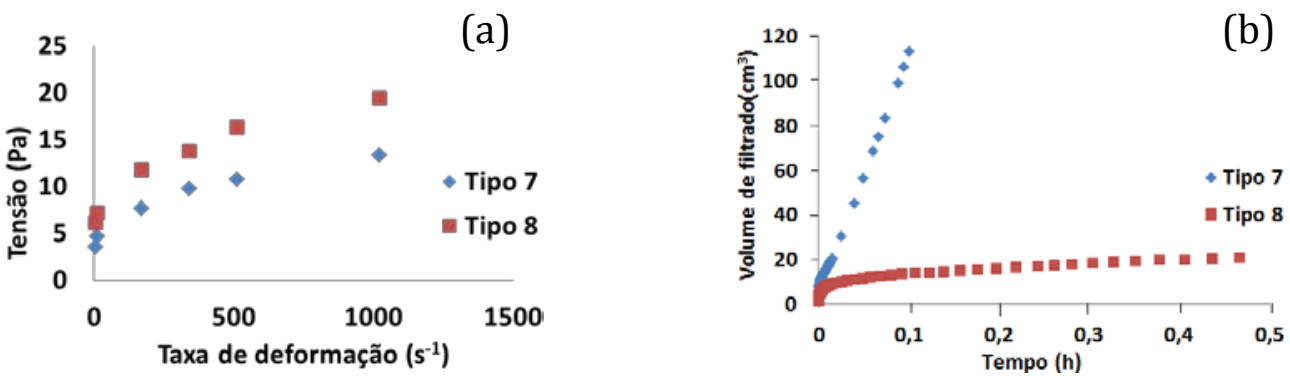

Figura 6- (a) Reograma dos fluidos analisados; (b) Curva de Filtração estática - Tipo 7 e 8 
Observando a Figura 6 (a) é possível verificar que a diferença do tamanho da partícula utilizada causa alteração no comportamento reológico do fluido. O experimento de filtração para o fluido (tipo 7) com esfera de vidro (-75 micra) foi realizado em apenas 6 minutos e com uma pressão de 500 psi, o baixo tempo de filtração ocorreu devido ao elevado volume de filtrado. Já para o fluido (tipo 8) com esfera de vidro (-300+180 micra) a filtração foi realizada durante 30 minutos, sendo 15 minutos a uma pressão de 500 psi e 15 minutos em 1000 psi. Isto se deve ao maior adensamento e consequente dificuldade de escoamento de fluido através da torta com esfera de vidro de maior granulometria, conforme pode ser visto na Figura 7a torta não consolidada formada pelo fluido Tipo 7 e Figura $7 \mathrm{~b}$ torta formada pelo fluido Tipo 8.
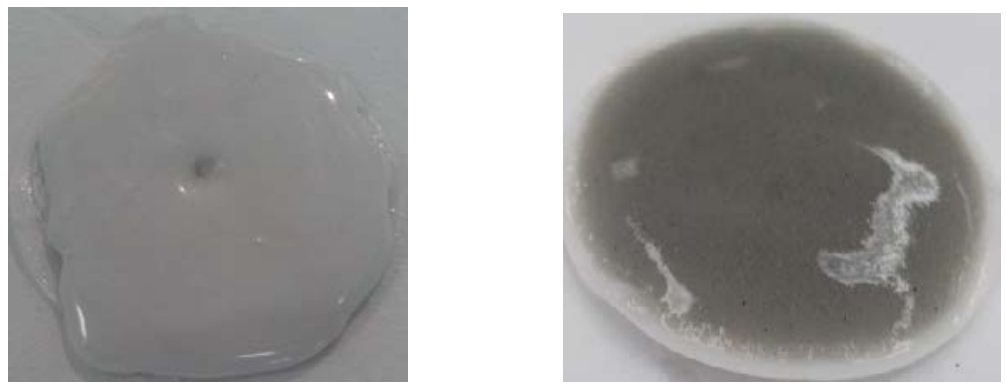

Figura 7- (a) Torta esfera de vidro -75 micra; (b) Torta esfera de vidro -300 +180 micra.

\subsubsection{Experimento com carbonato de cálcio e goma xantana}

Neste tópico desejou-se avaliar o efeito da interação de um sólido não inerte com diferentes faixas granulométricas sobre a reologia do fluido e o volume de filtrado. A Figura 8a mostra a reologia dos fluidos e Figura $8 \mathrm{~b}$ apresenta o volume de filtrado obtido em função do tempo de filtração.
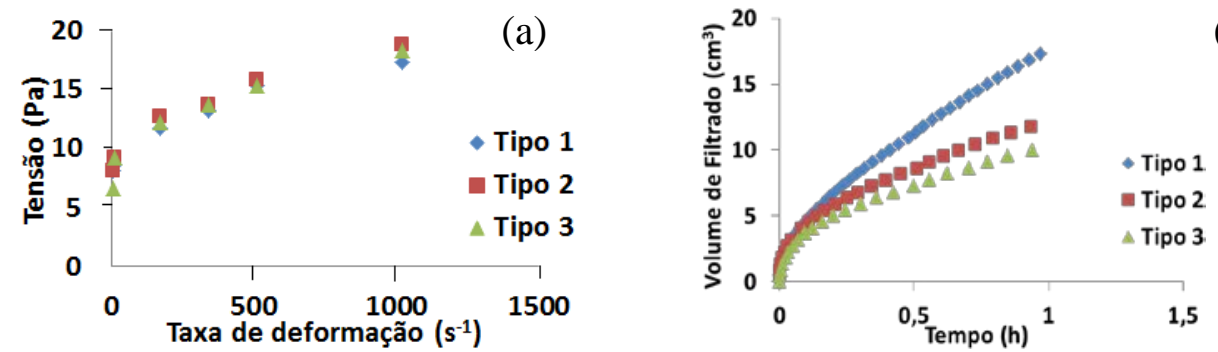

Figura 8- (a) Reograma dos fluidos avaliados. (b) Curva de Filtração estática - Tipo 1, 2 e 3

Analisando a Figura 8, pode-se verificar que o fluido Tipo 1 composto por partículas menores produz um maior volume de filtrado. Isto possivelmente se deve a uma interação entre a goma xantana e as partículas do carbonato de cálcio de menor diâmetro. Outro fator que pode estar atuando é o efeito do polímero goma xantana, que tem sua viscosidade aparente alterada com o aumento da pressão. 


\section{CONCLUSÃO}

Fluidos com a goma xantana o efeito distribuição de partículas, leva a uma interação entre as mesmas provocando um aumento no volume de filtrado com a diminuição do diâmetro da partícula. Mais testes serão realizados a fim de investigar o real efeito deste comportamento. Testes realizados com glicerina e esfera de vidro mostraram que o volume de filtrado aumentou com o aumento da distribuição granulometria da esfera. Já os fluidos com goma xantana, o efeito foi o mesmo que o obtido com carbonato de cálcio, mostrando a significancia do efeito da não-newtoneidade sobre o processo de filtração. Para os demais fluidos preparados ora com goma ou carbonato de cálcio os resultados foram contraditório a literatura, que afirma que o volume de filtrado é aumentado com o aumento da distribuição granulométrica. A diferença da granulometria do carbonato de cálcio selecionado alterou as propriedades reológicas dos fluidos avaliadas. Para todos os fluidos simulados, o volume de filtrado aumentou com o aumento da pressão de filtração.

Concluiu-se que a presença de polímeros viscosificante causa um aumento de filtrado com a diminuição do diâmetro das partículas. Resultados semelhantes foram obtidos com carbonato de cálcio e esfera de vidro.

\section{REFERÊNCIAS}

Bailey, L., BOEK, E.S., JACQUES, S.D.M, EL AL., 2000, Particle Invasion from Drilling Fluids, SPE 67853, SPE Journal, 5 Sept, 2001.

Calabrez, N. D. Filtração e Invasão de Fluidos de Perfuração: Estudo Comparativo, Caracterização da Torta e Modelagem, Dissertação de mestrado Universidade Federal Rural do Rio de Janeiro, 2013.

Chesser, B.G.; Clark, D.E.; Wise, W.V. Dynamic and Static Filtrate-Loss Techniques for Monitoring Filter-Cake Quality Improves Drilling-Fluid Performance, SPE Drilling \& Completion, New Orleans, September de 1994.

Civan, F.; Reservoir Formation Damage Fundamentals, Modeling, Assessment, and Mitigation, $2^{0}$ Edição, 2007.

Gallino, G.; Migliori, M.; Cindio, B. A Rheological Approach to Drill-in Fluids Optimization, Rheologica Acta, Vol. 40, p. 196-203, 2001.

Gates, G.L. and Bowie, C.P. Correlation of Certain Properties of Oil Well Drilling Fluids with Particle Size Distribution. Unite States Bureau of Mines, R.I. 3645, pp. 1-22, 1942.

Krumbein, W. C., and Monk, G. D. Permeability as a Function of the Size Parameters of Unconsolidated Sand, Trans AIME, vol. 151, pp. 153- 163, 1943 